\title{
Maximum thicknesses of EELS log ratio thickness measurement for several elements
}

Misa Hayashida ${ }^{1}$ and Marek Malac ${ }^{2}$

${ }^{1}$ National Research of Council, Edmonton, Alberta, Canada, ${ }^{2}$ National Research of Council, Alberta, Canada

Sample thickness $(t)$ projected along the electron beam path can be measured in a transmission electron microscope (TEM) equipped with an energy filter for example by the log-ratio method. The relation underlying the log-ratio method is

$t=\lambda \ln \left(\frac{I}{I_{0}}\right)(1)$

here $t, \lambda, I$ and $I_{0}$ are sample thickness, inelastic mean free path (IMFP), total intensity of the incident beam and zero-loss filtered intensity $[1,2]$. Eq. (1) can be applied to a pair of unfiltered and filtered images pixel by pixel providing a two-dimensional sample thickness map. We investigate the upper limit of the method when applied to thick samples. In particular, we are concerned with the maximum thickness yielding a linear dependence of $\ln \left(I / I_{0}\right)$ on $t$.

Aluminum and Epon exhibit linear response up to $\ln \left(I / I_{0}\right)<5$ at $200 \mathrm{kV}$ [3]. Al and Si linear dependence for $\ln \left(I / I_{0}\right)<\sim 1.2$, and Fe up to $\ln \left(I / I_{0}\right)<\sim 2.2$ at $200 \mathrm{kV}$ with collection semi-angle $(\beta) 11.9-35.7 \mathrm{mrad}$ was reported in [4]. $\mathrm{Ni}, \mathrm{Al}_{2} \mathrm{O}_{3}, \mathrm{Si}$ and $\mathrm{SiO}_{2}$ linear dependence were limited to $\ln \left(I / I_{0}\right)<0.5-0.6$ at 300 $\mathrm{kV}$ with $\beta=17.7-25.1 \mathrm{mrad}[5]$.

Alternatively, $t$ can be measured by convergent beam electron diffraction (CBED)[1, 6, 7], KramersKronig sum method [8,9], and SEM [10, 11] Diameter of rod-shaped samples fabricated by a focus ion beam (FIB) was also used to estimate $t$ at the center of rod-shaped samples[4, 5].

The maximum thickness $t_{\max }$ yielding linear response depends on collection semi-angle $\beta$ and on the sample atomic number $\mathrm{Z}$. We measured the relationships of $\ln \left(I / I_{0}\right)$-t for $\mathrm{SiO}_{2}, \mathrm{Al}, \mathrm{Si}, \mathrm{Ti}, \mathrm{Ni}, \mathrm{Cu}, \mathrm{Ag}$ and Au rectangular profile rod sample, see Fig. 1a, using a 300 kV Hitachi H9500 TEM with Gatan Tridiem ${ }^{\text {TM }}$ electron energy filter. Slice sections from reconstructed images by electron tomography were used to measure local projected $t$ precisely, see Fig. 1b. The dependence of $\ln \left(I / I_{0}\right)$ on $t$ with $\beta=16$ mrad are shown in Fig 2a.

Fig. $2 \mathrm{~b}$ shows plots of $t_{\max }$ extracted from Fig. 2a. The $t_{\max }$ decreases with increasing atomic number. The plots were fitted to $a+b^{*} \log (Z)$. Here, $Z$ is an atomic number, $\mathrm{a}$ and $\mathrm{b}$ are fit constants. Using this phenomenological fit, we it may be possible to predict $t_{\max }$ for other materials. The relationships the relationships of $\ln \left(I / I_{0}\right)-t$ and $t t_{\max }$ were also measured for each material with $\beta=3,5,9$ and 99 mrad.

(a)

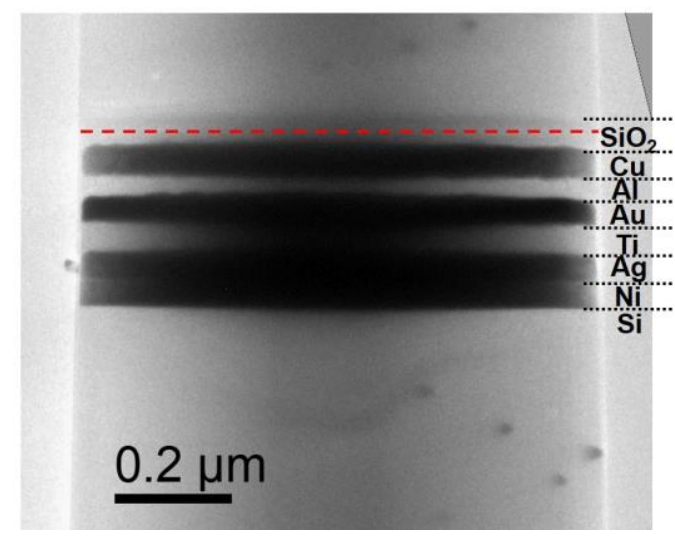

(b)

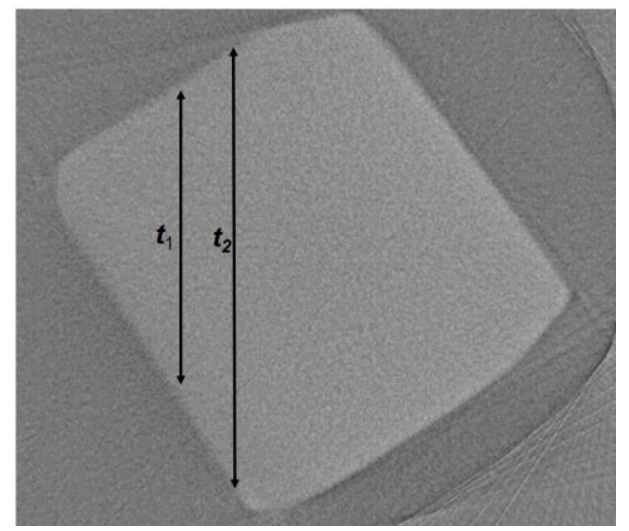


Figure 1. a) A TEM image of a rod-shaped multilayer sample fabricated by FIB. b) A slice section from a reconstructed 3D image of the sample in a). The slice section was extracted at location of the red dashed line in a). Two projected thicknesses $t_{1}$ and $t_{2}$ are indicated by arrows.

(a)
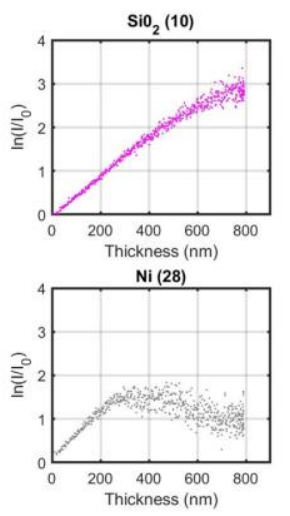

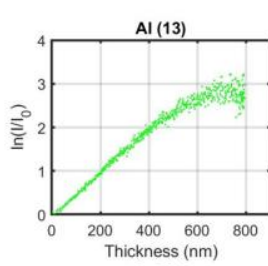

$\mathrm{Cu}(29)$

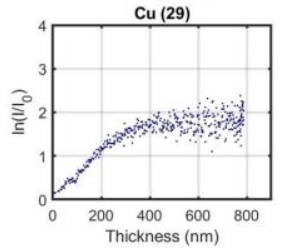

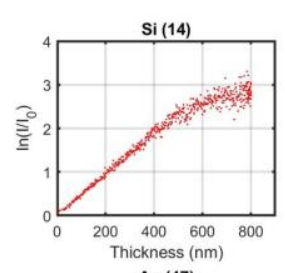

$\mathrm{Ag}(47)$

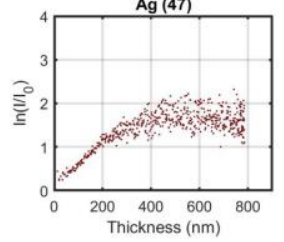

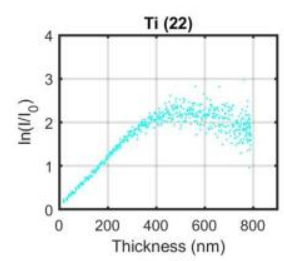

$\mathrm{Au}(79)$

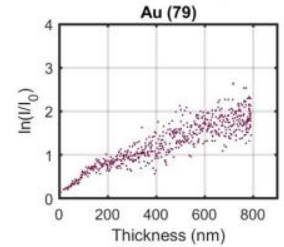

(b)

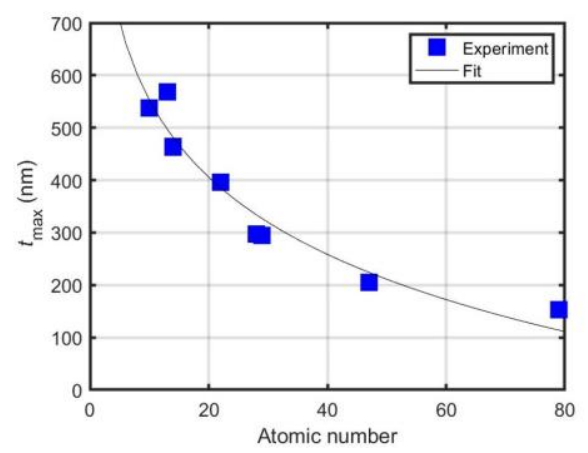

Figure 2. (a) Measured relationships of $\ln \left(I / I_{0}\right)$ vs. $t$ for $\mathrm{SiO}_{2}, \mathrm{Al}, \mathrm{Si}, \mathrm{Ti}, \mathrm{Ni}, \mathrm{Cu}, \mathrm{Ag}$ with $\beta=16 \mathrm{mrad}, t$ was extracted at multiple locations as indicated in Fig 1b. (b) Maximum thickness $t_{\max }$ yielding a linear dependence of $\ln \left(I / I_{0}\right)$ on $t$ from several element with $\beta=16 \operatorname{mrad}$ at $300 \mathrm{keV}$.

Reference

[1] T. Malis, S.C. Cheng, R.F. Egerton, EELS Log-ratio technique for specimen-thickness measurement in the TEM, Journal of Electron Microscopy Technique, 8 (1988) 193-200.

[2] R.F. Egerton, Electron Energy-Loss Spectroscopy in the Electron Microscope, 2 ed., Plenum Press, New York, 1996.

[3] J. Hosoi, T. Oikawa, M. Inoue, Y. Kokubo, K. Hama, Measurement of partial specific thickness (net thickness) of critical-point-dried cultured fibroblast by energy analysis, Ultramicroscopy, 7 (1981) 147153.

[4] K. Oh-Ishi, T. Ohsuna, Inelastic mean free path measurement by STEM-EELS technique using needleshaped specimen, Ultramicroscopy, 212 (2020) 112955.

[5] H. Meltzman, Y. Kauffmann, P. Thangadurai, M. Drozdov, M. Baram, D. Brandon, W.D. Kaplan, An experimental method for calibration of the plasmon mean free path, J. Microsc., 236 (2009) 165-173.

[6] P.L. Potapov, The experimental electron mean-free-path in Si under typical (S)TEM conditions, Ultramicroscopy, 147 (2014) 21-24.

[7] D.R.G. Mitchell, Determination of mean free path for energy loss and surface oxide film thickness using convergent beam electron diffraction and thickness mapping a case study using Si and P91 steel, Journal of Microscopy, 224 (2006) 187-196.

[8] K. Iakoubovskii, K. Mitsuishi, Y. Nakayama, K. Furuya, Thickness Measurements With Electron Energy Loss Spectroscopy, Microsc. Res. Tech., 71 (2008) 626-631. 
[9] K. Iakoubovskii, K. Mitsuishi, Mean free path of inelastic electron scattering in elemental solids and oxides using transmission electron microscopy: Atomic number dependent oscillatory behavior, PHYSICAL REVIEW B, 77 (2008) 104102.

[10] P. Zhang, Z. Wang, J.H. Perepezko, P.M. Voyles, Elastic and inelastic mean free paths of 200keV electrons in metallic glasses, Ultramicroscopy, 171 (2016) 89-95.

[11] D.T. Schweiss, J. Hwang, P.M. Voyles, Inelastic and elastic mean free paths from FIB samples of metallic glasses, Ultramicroscopy, 124 (2013) 6-12. 